\title{
Diabetes mellitus and thyroid diseases
}

\author{
Masahiro Nishi ${ }^{1}$
}

Received: 24 January 2018 / Accepted: 6 March 2018 / Published online: 9 March 2018

(C) The Japan Diabetes Society 2018

\begin{abstract}
Both diabetes mellitus and thyroid diseases are very common in the fields of endocrinology and metabolism. Coexistence of diabetes mellitus and thyroid diseases are frequently experienced. However, higher prevalence of thyroid dysfunction in both type 1 and type 2 diabetes compared with non-diabetics have been reported. Autoimmunity is a key to understand the link between type 1 diabetes and autoimmune thyroid diseases. And, this combination of autoimmune diseases is recognized as autoimmune polyglandular syndrome type 3 variant. On the other hand, the relation between thyroid function and glucose intolerance or type 2 diabetes mellitus is more complicated. Both hyperthyroidism and hypothyroidism could be risk factors for glucose intolerance. In this review, current knowledge about the relationship between diabetes mellitus and thyroid diseases is described, which includes the following topics: effects of thyroid hormone on glucose metabolism, subclinical hypothyroidism and diabetic complications, type 1 diabetes and autoimmune thyroid diseases, and several clinical pitfalls in patients with both diseases.
\end{abstract}

Keywords Type 1 diabetes mellitus - Type 2 diabetes mellitus $\cdot$ Autoimmune thyroid disease $\cdot$ Autoimmune polyglandular syndrome

\section{Introduction}

Diabetes mellitus is a systemic disease, and its clinical presentation is not restricted in pancreatic beta cells. Diabetic complications affect the whole body including retina, kidney, nervous system and vascular system. Similarly, since thyroid hormone is a key regulator of whole body metabolism and energy expenditure, thyroid dysfunction affect many organs of whole body far beyond a tiny cervical gland. Both diabetes mellitus and thyroid diseases are very common in the fields of endocrinology and metabolism. Coexistence of diabetes mellitus and thyroid diseases are frequently experienced. However, higher prevalence of thyroid dysfunction in both type 1 and type 2 diabetes have been reported [1,2]. Therefore, they are not simple bystanders, but there would

Part of this review was presented at Advances in Diabetology, the 51 st annual postgraduate course in Japan Diabetes Society held in Kyoto, Japan in 2017.

Masahiro Nishi

mnishi@wakayama-med.ac.jp

1 Division of Clinical Nutrition and Metabolism, Wakayama Medical University, 811-1 Kimiidera, Wakayama, Wakayama 641-8509, Japan be some clinical linkage between them. Autoimmunity is a key to understand the link between type 1 diabetes and autoimmune thyroid diseases. And, this combination of autoimmune diseases is recognized as autoimmune polyglandular syndrome type 3 variant. On the other hand, the relation between thyroid function and type 2 diabetes is more complicated. Both hyperthyroidism and hypothyroidism could be risk factors for glucose intolerance. This review summarizes the associations between diabetes mellitus and thyroid diseases, and discusses some clinical pitfalls in patients with both diabetes mellitus and thyroid diseases.

\section{Thyroid hormone and glucose metabolism}

Thyrotoxicosis is more common in type 2 diabetes mellitus than in general population. Thyroid hormone affects glucose metabolism via several organs (Table 1). Excess thyroid hormone causes oxyhyperglycemia (rapid increase of blood glucose after oral glucose load) by enhancing gastrointestinal mobility and glucose absorption. Enhancement of glucose absorption by thyroid hormone is supposed to be a result of increased gastrointestinal mobility; however, there might be a direct effect of thyroid hormone on 
Table 1 Effects of thyroid hormone on glucose metabolism in specific organ or cells

\begin{tabular}{ll}
\hline Organ & Effects on glucose metabolism \\
\hline Gastrointestinal tract & $\begin{array}{c}\text { Increase of mobility of gastrointestinal tract, Increased absorp- } \\
\text { tion of glucose } \\
\text { Increase of PEPCK activity, Increase of gluconeogenesis }\end{array}$ \\
Liver & Increase of lipolysis, elevation of serum free fatty acid \\
Adipose tissue & Increase of GLUT4, increase of glucose uptake \\
Skeletal muscle & Inhibition of apoptosis, activation of growth and differentiation \\
Pancreatic beta cells & Increase of insulin secretion \\
Pancreatic alpha cells & Increase of glucagon secretion \\
\hline
\end{tabular}

a
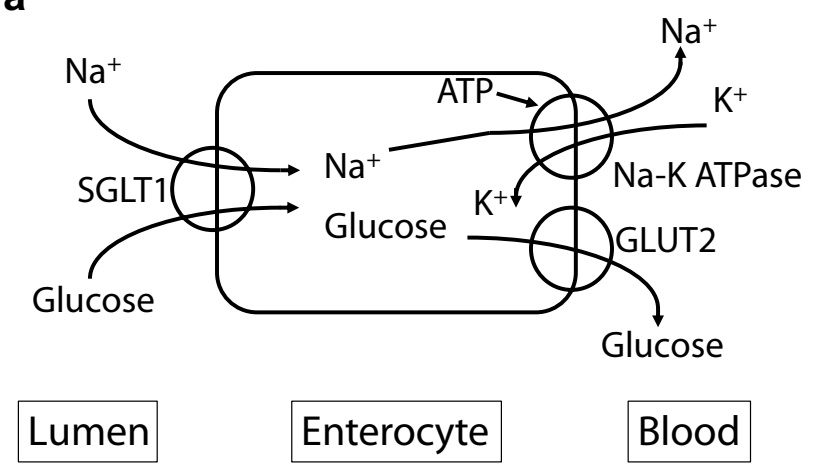

b

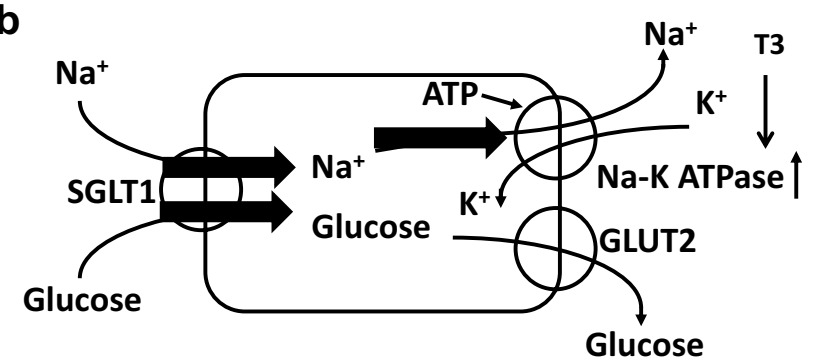

Lumen

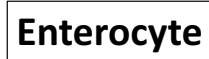

Fig. 1 Sodium/glucose cotransport in enterocyte. a Euthyroid state. SGLT1 expressed in luminal membrane cotransports sodium/glucose into enterocytes. Sodium or glucose is excreted into blood vessel via Na-K ATPase or GLUT2, respectively. b Hyperthyroid state. Since T3 activate Na-K ATPase, Na flux increased which causes enhancement of SGLT1 activity

gastrointestinal tract. T3 treatment enhances $\mathrm{Na}^{+}$glucose cotransporter (SGLT)-1 activity in human colon cancerderived enterocyte-like Caco-2 cells. Although SGLT-1 mRNA was increased by T3 treatment, SGLT-1 protein level did not change. Since thyroid hormone increases $\mathrm{Na}^{+}-\mathrm{K}^{+}$ ATPase activity, this enhancement of SGLT-1 activity was thought to be a result of $\mathrm{Na}^{+}$flux due to increased $\mathrm{Na}^{+}-\mathrm{K}^{+}$ ATPase activity by thyroid hormone (Fig. 1) [3]. Thyroid hormone also increases gluconeogenesis in liver. This effect is through direct effect of thyroid hormone or indirect effect via glucagon or catecholamine. In adipose tissues, thyroid hormone stimulates lipolysis, and increases serum free fatty acid level which causes insulin resistance. GLUT4 expression and glucose uptake in skeletal muscle are increased in hyperthyroidism. In pancreatic beta cells, thyroid hormone stimulates insulin secretion, although degradation of insulin is also enhanced by thyroid hormone. In pancreatic alpha cells, thyroid hormone enhances glucagon secretion. Via sum of these effects on glucose metabolism by thyroid hormone, excess thyroid hormone will deteriorate glucose metabolism and cause glucose intolerance or diabetes mellitus. Moreover, since thyroid hormone enhances lipolysis, hyperthyroidism is a risk of ketosis, if complicated with insulin deficient state. Even within normal range of thyroid hormone, thyroid hormone level was reported to be positively associated with insulin resistance in the early stage of type 2 diabetes [4].

\section{Subclinical hypothyroidism and diabetic complications}

Hypothyroidism is not only a risk of obesity and dyslipidemia, but that of metabolic syndrome and diabetes mellitus. As already mentioned, excess thyroid hormone causes insulin resistance and glucose intolerance. Lack of thyroid hormone also associated with insulin resistance and glucose intolerance, and treatment of hypothyroidism was reported to improve insulin sensitivity $[1,2,5]$. Recent review and meta-analysis revealed that patients with type 2 diabetes mellitus were associated with a 1.93-fold increase in a risk of subclinical hypothyroidism compared to non-diabetics [6]. And moreover, diabetic complications were more prevalent in patients with both type 2 diabetes mellitus and subclinical hypothyroidism than those with type 2 diabetes mellitus of normal thyroid function [6]. The odds ratio for diabetic nephropathy was 1.74 (95\% confidence interval 1.34-2.28), that for diabetic retinopathy was 1.42 (95\% confidence interval 1.21-1.67), that for diabetic peripheral neuropathy was 1.87 (95\% confidence interval 1.06-3.28), and that for peripheral arterial disease was 1.85 (95\% confidence interval 
1.35-2.54). And, overall odds ratio for diabetic complications was 1.74 (95\% confidence interval $1.34-2.28)$. The precise mechanism of this aggravating effect of subclinical hypothyroidism on diabetic complications is not fully clarified. Patients with subclinical hypothyroidism may have decreased cardiac output, renal flow, glomerular filtration and increased vascular resistance [7]. These changes may explain the higher prevalence of diabetic nephropathy in patients with subclinical hypothyroidism. As for diabetic retinopathy, thyroid hormone is also important for the development of the retina. Hypothyroid rat had smaller and thinner retina [8]. In terms of macrovascular complications, patients with hypothyroidism have been reported higher incidence of dyslipidemia and increased intima-media thickness [9]. Furthermore, hypothyroidism may be related with increased vascular stiffness, endothelial dysfunction, and hypercoagulability. All of these factors could contribute the higher prevalence of peripheral arterial disease in diabetic patients with subclinical hypothyroidism.

\section{Type 1 diabetes mellitus and autoimmune thyroid diseases}

Type 1 diabetes mellitus and autoimmune thyroid diseases including Graves' disease and Hashimoto thyroiditis are autoimmune diseases and share common etiology. Therefore, type 1 diabetes mellitus and autoimmune thyroid diseases are complicated with other autoimmune diseases with higher incidence. Autoimmune polyglandular syndrome (APS) is a syndrome with multiple endocrine organ defects because of autoimmune etiology which is classified into 4 types according to Neufeld and Blizzard [10] (Table 2). APS type 1, also called as APECED (autoimmune polyendocrinopathy-candidiasis-ectodermal dystrophy), is inherited in an autosomal recessive fashion, and the gene responsible for APS type 1 was identified as AIRE (autoimmune regulator) located on chromosome 21q22.3. AIRE gene product is a transcriptional factor mainly expressed in medullary thymic epithelial cells where self-tolerance is provided. Therefore, mutations in the AIRE gene will destroy this self-tolerance mechanism and lead to autoimmunity. Clinically, APS type 1 is characterized by 3 components: chronic candidiasis, chronic hypoparathyroidism, and autoimmune Addison's disease. APS type 2, also known as Schmidt's syndrome, is multifactorial etiology and links to HLA. Clinical presentation of APS type 2 is characterized by the presence of autoimmune Addison's disease in association with either autoimmune thyroid diseases and/or type 1 diabetes mellitus. Combination of type 1 diabetes mellitus and autoimmune thyroid diseases without autoimmune Addison's disease is classified into APS type 3, and this specific combination of type 1 diabetes mellitus and autoimmune thyroid diseases without other autoimmune diseases is also called as APS type 3 variant (3v). In Japanese people, the autoimmune diseases mostly complicated with type 1 diabetes mellitus are the autoimmune thyroid diseases. Comparison of clinical characteristics of 54 Japanese APS $3 \mathrm{v}$ patients and 143 type 1 diabetic patients without autoimmune thyroid diseases were reported [11]. APS $3 \mathrm{v}$ patients showed female predominance, slow and older onset, and higher GAD antibody positivity. And, the analysis of HLA haplotype and CTLA4 gene polymorphism revealed some differences in genetic background between these two. APS type 4 is defined as combination of autoimmune diseases other than type $1-3$.

\section{Clinical pitfalls in patients with both diabetes mellitus and thyroid disease}

\section{Glycated albumin}

Along with glycated hemoglobin, glycated albumin is also used as an indicator of glycemic control in diabetic patients. Since glycated albumin is affected not only by glycemic control but by albumin metabolism, and since thyroid hormone promotes albumin catabolism, patients with hypothyroidism show higher serum glycated albumin level and those with hyperthyroidism show lower. Significant positive correlation between serum glycated albumin and TSH levels, and inverse correlation between serum glycated albumin and free T3 or free T4 levels was reported [12]. Therefore, careful assessment of serum glycated albumin levels should be needed in diabetic patients with thyroid dysfunction.

Table 2 Classification of autoimmune polyglandular syndrome according to Neufeld and Blizzard

\begin{tabular}{lc}
\hline APS type 1 & Chronic candidiasis, chronic hypoparathyroidism, autoimmune Addison's disease (at least two present) \\
\hline APS type 2 & Autoimmune Addison's disease + autoimmune thyroid disease and/or type 1 diabetes mellitus \\
APS type 3 & $\begin{array}{c}\text { Autoimmune thyroid disease + other autoimmune diseases (excluding Addison's disease, hypoparathy- } \\
\text { roidism, chronic candidiasis) }\end{array}$ \\
APS type 4 & Two or more organ-specific autoimmune diseases (which do not fall into type 1, 2, or 3)
\end{tabular}




\section{Seaweed, especially Kombu or kelp}

In the diet therapy planned for diabetic patients, intake of seaweed is highly recommended, because it contains few calories and is rich in minerals and dietary fiber. However, it contains lots of iodine, especially Kombu or kelp does. Excess iodine intake may cause thyroid dysfunction in susceptible patients, such as patients with autoimmune thyroid diseases.

\section{Methimazole and insulin autoimmune syndrome}

Methimazole is a widely used antithyroidal drug in the treatment for Graves' disease. Since it contains sulfhydryl group, the use of methimazole is related to the development of insulin autoimmune syndrome [13]. As a cause of hypoglycemia in patients with diabetes mellitus and Graves' disease treated with methimazole, it is necessary to take into account the possibility of insulin autoimmune syndrome.

\section{Metformin and thyroid diseases}

Metformin is a widely used antidiabetic drug. Recently, it has been reported that serum TSH level was decreased by metformin therapy [14-16]. Several hypotheses have been proposed to explain TSH-lowering effects of metformin. The possibility that metformin might increase thyroid hormone absorption from gastrointestinal tract is not likely, since serum free thyroid hormone levels were unchanged. Since metformin crosses blood-brain barrier, central mechanism of TSH suppression is another explanation. Although metformin activates adenosine-5'-monophosphate-activated kinase (AMPK) in peripheral tissues, metformin was reported to inhibit AMPK in the hypothalamus [17]. This central action of metformin may be involved in its TSH suppression. However, further studies would be necessary to elucidate the mechanism of TSH-lowering effects of metformin. Metformin's effects on thyroid are not limited in TSH lowering action. Diabetic patients have been reported to have increased thyroid volume and higher prevalence of thyroid nodules. Patients with metformin treatment had smaller thyroid volumes and lower risk of thyroid nodules compared with controls. Although precise mechanism was not fully elucidated, some studies demonstrated that metformin can inhibit the growth of thyroid cancer cells by affecting insulin/insulin-like growth factor I signal and mammalian target of rapamycine pathways $[18,19]$.

\section{Thiazolidinediones (TZD) and thyroid-associated orbitopathy}

The pathogenesis of thyroid-associated orbitopathy has not been fully clarified. However, it is believed to be an autoimmune inflammatory process and the expansion of the volume of orbital contents occur which includes fat and connective tissues as well as ocular muscles. This expansion of adipose tissues in thyroid-associated orbitopathy may be due to de novo adipogenesis. Orbital fibroblasts are capable to differentiate into adipocytes under appropriate stimuli, possibly inflammatory cytokines. One of the important factors in adipocyte differentiation is peroxisome proliferator-activated receptor (PPAR)-gamma which is expressed in orbital adipose and connective tissues and increased expression in active stages of thyroid-associated orbitopathy was reported [20, 21]. TZD, PPAR-gamma agonist, is an antidiabetic drug of insulin-sensitizing effects. The administration of TZD to patients with thyroid-associated orbitopathy has been reported to associate with aggravation of the ophthalmopathy [22].

\section{Glucagon-like peptide-1 (GLP-1) receptor agonist and thyroid cancer}

GLP-1 receptor agonists are increasingly being used for the treatment of type 2 diabetes mellitus. While GLP-1 receptor agonists can efficiently reduce blood glucose, they may potentially have some effects on thyroid gland, since there exist GLP-1 receptors. There are four types of thyroid cancer: papillary, follicular, anaplastic and medullary thyroid cancers. Medullary thyroid cancer is a very rare and it is derived not only from thyroid follicular cells, but also from thyroid C-cells which express and secrete calcitonin. Since GLP-1 receptor agonists have been reported to activate rodent thyroid C-cells and cause calcitonin release and C-cell proliferation [23], they are currently contraindicated in patients with medullary thyroid cancer and in patients with multiple endocrine neoplasia type 2 which is a genetically predisposed condition to medullary thyroid cancer. However, compared to rodents, primates or human have fewer GLP-1 receptors in thyroid C-cells [23, 24]. Especially normal human C-cells do not express GLP-1 receptor at all, while $27 \%$ of human medullary thyroid cancers express GLP-1 receptor and $100 \%$ of rat C-cell hyperplasia or medullary thyroid cancer does. And furthermore, the use of GLP-1 receptor agonists did not lead to elevation of serum calcitonin levels in human [25], although long-term observation may be necessary. Therefore, at present the benefits of using GLP-1 receptor agonist for patients with type 2 diabetes mellitus overwhelmingly outweigh its possible risks except for patients with medullary thyroid cancer or multiple endocrine neoplasia (MEN) type 2.

\section{Conclusion}

Since diabetes mellitus and thyroid diseases are very common diseases, they may complicate each other very frequently. However, higher prevalence of thyroid dysfunction 
in both type 1 and type 2 diabetes compared with non-diabetics revealed their close relationship. Of course, autoimmunity is a key to understand the link between type 1 diabetes and autoimmune thyroid diseases. On the other hand, both hyperthyroidism and hypothyroidism have been correlated with type 2 diabetes mellitus. Higher incidence of diabetic complications has also been reported in subclinical hypothyroidism. We have to pay some special attention to patients with both diabetes mellitus and thyroid diseases, such as assessment of glycated albumin, iodine uptake, and treatment drugs.

\section{Compliance with ethical standards}

Conflict of interest The author declares there are no conflicts of interest.

Research involving human participants and/or animals There are no studies with human subjects and/or animals performed by author.

\section{References}

1. Chaker L, Ligthart S, Korevaar TIM, Hofman A, Franco OH, Peeters RP, Dehghan A. Thyroid function and risk of type 2 diabetes: a population-based prospective cohort study. BMC Med. 2016;14:150-7.

2. Duntas LH, Orgiazzi J, Brabant G. The interface between thyroid and diabetes mellitus. Clin Endocrinol. 2011;75:1-9.

3. Matosin-Matekalo M, Mesonero JE, Delezay O, Poiree JC, Ilundain AA. Thyroid hormone regulation of the $\mathrm{Na}^{+} /$glucose cotransporter SGLT1 in Caco-2 cells. Biochem J. 1998;334:633-40.

4. Lambadiari V, Mitrou P, Maratou E, Raptis AE, Tountas N, Raptis SA, Dimitriadis. Thyroid hormones are positively associated with insulin resistance early in the development of type 2 diabetes. Endocrine. 2011;39:28-32.

5. Joffe BI, Distiller LA. Diabetes mellitus and hypothyroidism: strange bedfellows or mutual companions? World J Diabetes. 2014;5:901-4.

6. Han C, He X, Xia X, Li Y, Shi X, Shan Z, Teng W. Subclinical hypothyroidism and type 2 diabetes: a systematic review and meta-analysis. PLoS ONE. 2015. https://doi.org/10.1371/journ al.pone.0135233.

7. Iglesias P, Bajo MA, Selgas R, Diez JJ. Thyroid dysfunction and kidney disease: an update. Rev Endocrinol Metab Disord. 2017;18:131-44

8. Sevilla-Romero E, Munoz A, Pinazo-Duran MD. Low thyroid hormone levels impair the perinatal development of the rat retina. Ophthalmic Res. 2002;34:181-91.

9. Monzani F, Caraccio N, Kozakowa M, Dardano A, Vittone A, Taddei S, Palombo C, Ferrannini E. Effect of levothyroxine replacement on lipid profile and intima-media thickness in subclinical hypothyroidism: a double-blind, placebo-controlled study. J Clin Endocrinol Metab. 2004:89:2099-106.

10. Neufeld M, Blizzard RM. Polyglandular autoimmune diseases. In: Pinchera A, Doniach D, Fenzi GF, Baschieri L, editors.
Symposium on autoimmune aspects of endocrine disorders. New York: Academic Press; 1980. p. 357-65.

11. Horie I, Kawasaki E, Ando T, Kuwahara H, Abiru N, Usa T, Yamasaki H, Ejima E, Kawakami A. Clinical and genetic characteristics of autoimmune polyglandular syndrome type 3 variant in the Japanese population. J Clin Endocrinol Metab. 2012;97:E1043-50.

12. Koga M, Murai J, Saito H, Matsumoto S, Kasayama S. Effects of thyroid hormone on serum glycated albumin levels: study on non-diabetic subjects. Diabetes Res Clin Pract. 2009;84:163-7.

13. Uchigata $Y$, Hirata $Y$, Iwamoto $Y$. Insulin autoimmune syndrome (Hirata disease): epidemiology in Asia, including Japan. Diabetol Int. 2010;1:21-5.

14. Vigersky RA, Filmore-Nassar A, Glass AR. Thyrotropin suppression by metformin. J Clin Endocrinol Metab. 2007;91:225-7.

15. Meng X, Xu S, Chen G, Derwahl M, Liu C. Metformin and thyroid disease. J Endocrinol. 2017;233:R43-51.

16. Pappa T, Alevizaki M. Metformin and thyroid: an upadate. Eur Thyroid J. 2013;2:22-8.

17. Chau-Van C, Gamba M, Salvi R, Gaillard RC, Pralong FP. Metformin inhibits adenosine- 5'-monophospate-activated kinase activation and prevents increase in neuropeptide $\mathrm{Y}$ expression in cultured hypothalamic neurons. Endocrinology. 2007;148:507-11.

18. Chen G, Xu S, Renko K, Derwahl M. Metformin inhibits growth of thyroid carcinoma cells, suppresses self-renewal of derived cancer stem cells, and potentiate the effect of chemotherapeutic agents. J Clin Endocrinol Metab. 2012;97:E510-20.

19. Klubo-Gwiezdzinska J, Jensen K, Costello J, Patel A, Hoperia V, Bauer A, Burman KD, Wartofsky L, Vasko V. Metformin inhibits growth and decreases resistance to anoikis in medullary thyroid cancer cells. Endocr Relat Cancer. 2012;19:447-56.

20. Alevizaki M, Mantzou E, Cimponeriu A, Saltiki K, Philippou G, Wilersinga W. The Pro12Ala PPARgamma gene polymorphism: possible modifier of the activity and severity of thyroid-associated orbitopathy (TAO). Clin Endocrinol. 2009;70:464-8.

21. Starkey K, Heufelder A, Baker G, Joba W, Evans M, Davies S, Ludgate M. Peroxisome proliferator-activated receptor-gamma in thyroid eye disease: contraindication for thiazolidinedione use? J Clin Endocrinol Metab. 2003;88:55-9.

22. Miura LY, Villares SMF, Monteiro MLR, Guazzelli IC, Bloise W. Peroxisome proliferator-activated receptor-gamma gene expression in orbital adipose/connective tissues is increased during the active stage of Graves' ophthalmopathy. Thyroid. 2003;13:845-50.

23. Knudsen LB, Madsen LW, Andersen S, Almholt K, de Boer AS, Drucker DJ, Gotfredsen C, Egerod FL, Hegelund AC, Jacobsen H, Jacobsen SD, Moses AC, Molck A-M, Nielsen HS, Nowak J, Solberg H, Thi TDL, Zdravkovic M. Glucagon-like peptide-1 receptor agonists activate rodent thyroid $\mathrm{C}$-cells causing calcitonin release and C-cell proliferation. Endocrinology. 2010;151:1473-86.

24. Waser B, Beetschen K, Pellegata NS, Reubi JC. Incretin receptors in non-neoplastic and neoplastic thyroid $\mathrm{C}$-cells in rodents and humans: relevance for incretin-based diabetes therapy. Neuroendocrinology. 2011;94:291-301.

25. Hegedus L, Moses AC, Zdravkovic M, Thi TL, Daniels GH. GLP-1 and calcitonin concentration in humans: lack of evidence of calcitonin release from sequential screening in over 5000 subjects with type 2 diabetes or nondiabetic obese subjects treated with the human GLP-1 analog, liraglutide. J Clin Endocrinol Metab. 2011;96:853-60. 